\title{
Ischemia-modified albumin (IMA) in differential diagnosis of transient myocardial ischemia from non ischemic chest pain
}

\author{
Sokhanvar $\mathrm{S}^{1}$, Mellati $\mathrm{AO}^{1}$, Mousavinasab $\mathrm{SN}^{1}$, Taran L ${ }^{1}$, Vahdani $\mathrm{B}^{1}$, Golmmohamadi $\mathrm{Z}^{2}$
}

Department of Cardiology, Zanjan University of Medical Sciences, Zanjan, Iran. rozag2001@yahoo.com

\begin{abstract}
Background: Early diagnosis of acute coronary syndrome (ACS) is an important factor in reducing mortality of this disease. Cardiac troponins are not elevated within first hours. So there is a need to optimize the clinical applicability and accuracy of novel ACS markers, particularly with regard to utilizing this technique in combination with other diagnostic methods.

Methods: In this prospective study, we examined 226 patients between July 2009 and March 2010, admitted with chest pain to emergency room (ER). The study groups constisted of 120 subjects presenting with chest pain whose initial and subsequent diagnosis was unstable angina (UA), and 106 subjects whose initial diagnosis was unstable angina but subsequent diagnosis was non ischemic chest pain(NICP). For each patient electrocardiogram (ECG), cardiac troponins (cTnT), creatinine phosphokinase (CPK), IMA levels were measured. We used McNemar's test for correlated proportions and logistic regression and ROC curve for achieving better result.

Results: In this study median IMA values were definitely higher in patients with ACS compared with non ischemic chest pain (NICP) $(p<0.0001)(83.5$ to 49.6). An IMA cut-off threshold derived from the receiver operating characteristics curve (ROC) was $85 \mathrm{U} / \mathrm{ml}$ and gives $54 \%(95 \% \mathrm{Cl} 51$ to 56$)$ sensitivity and $87 \%(95 \% \mathrm{Cl} 83$ to 92) specificity in our population. Negative predictive value was $62 \%(95 \% \mathrm{Cl} 59$ to 66$)$. When IMA and ECG and CTnT were considered together sensitivity was $97.5 \%$ and specificity was $63 \%$, respectively.

Conclusion: Ischemia-modified albumin did not provide superior sensitivity or specificity compared with other diagnostic tests (Tab. 1, Fig. 2, Ref. 25). Full Text in PDF www.elis.sk.

Key words: ischemia modified albumin, acute coronary syndrome, unstable angina.
\end{abstract}

Early diagnosis of impaired myocardial perfusion before the occurrence of necrosis remains difficult for patients seen in the emergency department with symptoms of acute chest pain (1-3). Although ACS is often ruled out, this imposes a substantial financial burden and inconvenience to the patient, and medical system (4-6). The usual biomarkers (i.e, troponin, myoglobin) are sensitive and specific for the detection of myocardial necrosis, but show a greater rise approximately 3 to 6 hours after the onset of myocardial cell injury (7-8). Recently three markers have been proposed: choline, free fatty acids, and IMA (9). Currently form these markers only IMA is available as a licensed test (was registered by the United States Food and Drug Administration for the detection of cardiac ischemia $(10,11)$ for routine clinical application measured using the Albumin Cobalt Binding (ACB) assay (12). Ischemia modified albumin rises within minutes of myocardial ischemia onset and returns to baseline in 6 hours of restoring perfusion. Human serum albumin (HAS) has the capacity to bind certain transition metal ions, specially cobalt and copper, at the

${ }^{1}$ Department of Cardiology, Zanjan University of Medical Sciences, Zanjan, and ${ }^{2}$ Cardiovascular Research Center, Tabriz University of Medical Sciences, Tabriz, Iran

Address for correspondence: Z. Golmmohamadi, Cardiovascular Research Center, Tabriz University of Medical Sciences, Zanjan, Iran. Phone: +989143068423, Fax: +984113373919

Acknowledgement: Authors are thankful to Zanjan Medical University for financial support.
$\mathrm{N}$-terminus. The N-terminal (13) portion of HAS is susceptible to biochemical degradation and is less stable than the albumin of other species (14). IMA is a form of HAS in which the N-terminal amino acids are unable to bind to transition metals. Myocardial ischemia produces free radicals $(15,16)$. Acidosis develops and release of free iron and copper ions takes place (17). HSA is a hunter for divalent metal ions. It may thus be claimed that in ischemia, these processes may result in change in the ability of the $\mathrm{N}$-terminus to bind to transition metal ions. Our aim in this study is to evaluate the clinical performance of a new marker of ischemia, IMA alone and in combination with the other cardiac markers such as ECG, CPK, cTnT for early diagnosis of unstable angina (UA).

\section{Methods and materials}

This study was performed at Musavi Hospital, Zanjan, Iran and was approved by the local ethics committee. We engaged, on a prospective basis, patients who arrived at the ER within three hours of acute chest pain. However the enrolment period was between July 2009 and March 2010. All recruited patients had blood samples and an ECG in two hours of entrance, and received routine institutional care blinded to the IMA results. Standardized clinical data such as any history of coronary disease (like myocardial infarction, coronary bypass, angioplasty...), time of onset of acute chest pain, duration of symptoms, pattern of distribution, alteration of severity of pain were collected in special prepared ques- 
tionnaires at the ER. IMA, CPK and cTnT testing was carried out before any heparin/thrombolytic treatment was initiated. The excluded patients from our study were all of pregnant women, acute renal failure, patients with diagnosis of acute mesenteric ischemia, peripheral vascular disease, or any brain ischemia (13). The definition of UA is largely based on the clinical presentation. However in our study according to the Heas Chan (18) and Braunwald (19) references we introduced patients as UA with at least one of three features of chest pain: (1) Occurring at rest (or minimal exertion) and usually lasting $>20$ minutes (if not interrupted by Nitroglycerin administration); (2) being severe and of new onset (within 1 month) and (3) occurring with a crescendo pattern (more severe, prolonged, or frequent than previously). On the other hand patients were assessed as NICP when (1) a result of non cardiac mechanism was confirmed as the cause of chest pain; (2) all of these criteria were acquired: negative cTnT findings on serial sampling (over a 6-9 hour interval), presence of normal ECGs, and absence of any recent changes (lesions $<50 \%$ diameter reduction in any major epicardial vessel) or previous evidence of coronary artery disease; (3) a diagnosis of ACS was objectively ruled out after admission to the coronary cardiac unit (CCU).

\section{ECG classification}

Positive ECGs were those with ST segment depression $\geq 0.1$ $\mathrm{mV}$, or T wave inversion $\geq 0.2 \mathrm{mV}$ (in $\geq$ two contiguous leads) or left bundle branch block. ECGs that did not show any above criteria were considered negative (11). Each ECG was evaluated by the medical consulting physician of the ER and cardiac specialist as per routine institutional practice.

\section{Angiography and exercise tolerance test (ETT)}

Coronary angiography was performed in 36 patients, all procedures of angiography were carried out by an expert cardiologist blinded to the patient's clinical features and biochemistry results. A positive angiogram was defined as stenosis $\geq 50 \%$ diameter reduction in any main epicardial vessel. While exercise tolerance was accomplished in 46 patients and a specialist blindly evaluated the changes of it, divided to normal groups and negative ones. The cardiologists were responsible for assigning a final diagnosis based on the history, clinical examination, cTnT results and the data from perfusion scan, ETT, and coronary angiography, as available. However results of all findings were reviewed blinded to serum levels of IMA.

\section{Albumin cobalt binding test}

IMA was measured by the automated albumin cobalt binding assay (Miura-Italy $\mathrm{Co}$ ) run in accordance with manufactures instructions and the analyzer used was indicated. Blood was assembled for the IMA test in serum separated tubes and specimens like the way of Bar-Or D (20) were frozen at $-20^{\circ} \mathrm{C}$ within 2 hours. Frozen samples were slowly addied after liquefying. In the ACB Test, $95 \mu 1$ sample and $5 \mu 1$ cobalt chloride (coII) (Sigma Alorich, Germany co) were incubated for five minutes. During incubation, the (coII) attaches to the $\mathrm{N}$-terminus of unchanged albumin in the sample. The altered $\mathrm{N}$-terminal portion of albumin (was explained that occurred during ischemia) has lower tendency to attach to (coII). After incubation, $25 \mu$ dithiothreitol (DTT), (Merk, Germany co) is added to the combination. So DTT produces a colored complex with (coII) that is not bind at the $\mathrm{N}$-terminus of albumin, and this complex is measured with spectrophotometry at $492 \mathrm{~nm}$. In our laboratory, inter and intra assay coefficients of variation (CV) for the assay were $2.5 \%$ and $2.1 \%$, respectively. IMA values $\geq 85$ $\mathrm{u} / \mathrm{ml}$ were considered positive for cardiac ischemia in our study.

\section{Cardiac troponin $T$ test and creatine phosphokinase}

cTnT and CPK were assessed from serum separated tubes and cTnT concentration $\geq 0.05 \mathrm{ng} / \mathrm{ml}$ were considered positive. $\mathrm{cTnT}$ concentration were determined by Ramp instrument Ramp kits (Responsebio, Canada co) and CPK were measured by enzymatic assay with Pars Azmoon kits (Iran co), that concentrations $\geq 200$ $\mathrm{Iu} / \mathrm{ml}$ were considered as positive for us.

\section{Statistical analysis}

Results of IMA, ECG, and cTnT were investigated for clinical sensitivity, specificity, positive predictive value (PPV), and negative predictive value (NPV), alone and in combination. We considered positive when only one of the tests in combination study of IMA, ECG, and cTnT was positive. We used McNemar s test for correlated proportions and logistic regression and ROC curve for achieving better result. All statistical tests were performed using SPSS (16.5).

\section{Results}

A total of 237 patients were enrolled in to the study but 11 patients were left out from the final analysis as a result of clotting of blood samples or inexactness of timing for sample gaining. Therefore the total enrolled patients consisted of 226 patients, 122 men and 104 women with the mean age of 64 years (range of 29-88); 22 (9.7\%) had the history of myocardial infarction, 16 (7.1\%) had CABG and 23 (10.2\%) had previous angioplasty. Of

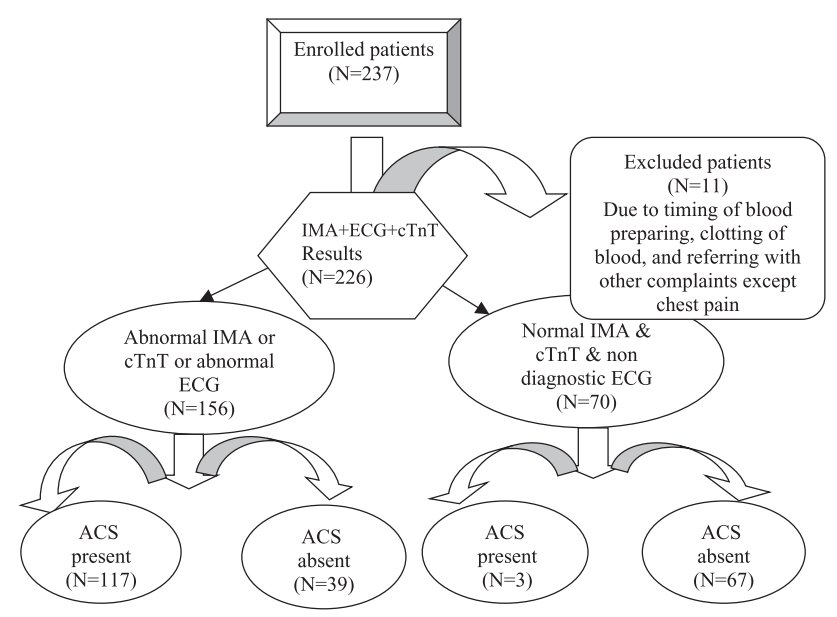

Fig. 1. Flowchart of diagnostic accuracy of IMA, cardiac troponin, \& ECG used together. 
Tab. 1. Presentation diagnostic tests for diagnosis of ACS.

\begin{tabular}{|c|c|c|c|c|c|c|c|c|c|}
\hline \multirow[t]{2}{*}{ Test } & \multicolumn{2}{|c|}{ Sensitivity for ACS } & \multicolumn{2}{|c|}{ Specificity for ACS } & \multicolumn{2}{|c|}{ PPV for ACS } & \multicolumn{2}{|c|}{ NPV for ACS } & \multirow{2}{*}{$\begin{array}{c}\text { ROC area } \\
\text { Under curve }\end{array}$} \\
\hline & $\%$ & $\mathrm{CI}$ & $\%$ & $\mathrm{CI}$ & $\%$ & $\mathrm{CI}$ & $\%$ & $\mathrm{CI}$ & \\
\hline IMA & 54 & 51 to 56 & 87 & 83 to 92 & 82 & 78 to 88 & 62 & 59 to 66 & 0.73 \\
\hline ECG & 88 & 83 to 93 & 95 & 91 to 98 & 79 & 73 to 85 & 85 & 79 to 90 & 0.79 \\
\hline CTnT & 14 & 9 to 20 & 74 & 68 to 81 & 77 & 72 to 83 & 47 & 45 to 51 & 0.54 \\
\hline ECG \& cTnT & 88 & 81 to 93 & 65 & 62 to 67 & 76 & 69 to 82 & 81 & 77 to 85 & 0.79 \\
\hline IMA \& cTnT & 48 & 45 to 51 & 85 & 79 to 90 & 80 & 76 to 85 & 57 & 55 to 60 & 0.70 \\
\hline IMA \& ECG & 97.5 & 94 to 99 & 64.2 & 60 to 67 & 75 & 69 to 81 & 95 & 91 to 98 & 0.80 \\
\hline
\end{tabular}

Total number of patients $=226$; total positive for $\mathrm{ACS}=131$; total negative for $\mathrm{ACS}=77$.

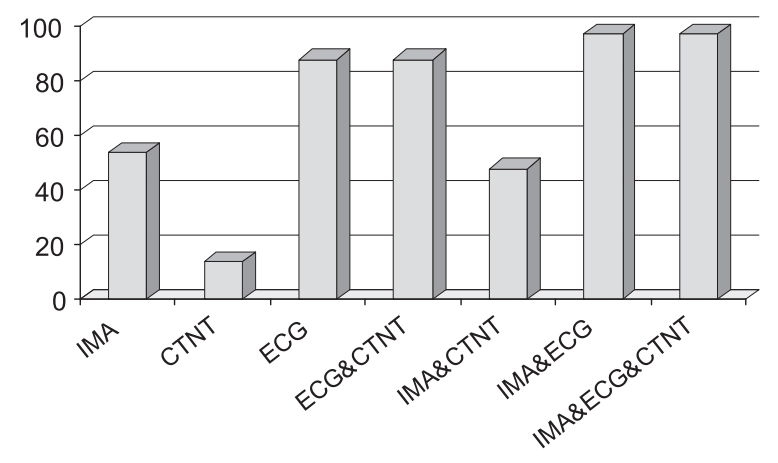

Fig. 2. Comparative sensitivities for diagnosis of ACS.

the 226 enrolled patients, 120 were discharged with a final diagnosis of ACS and 106 with the diagnosis of non ischemic chest pain. Among the ACS patients, we had 22 with NSTEMI, and 98 with UA. Of these, 29 had coronary stenosis on angiography and 8 patients had problem in ETT, and only 1 diagnosed with perfusion scan, the remaining patient had a history highly suggestive of UA. Of the 106 patients with a final diagnosis of NICP, a definite non cardiac cause of chest pain was identified in 80 . Among the 46 remained patients, 7 who underwent angiography had normal coronary arteries or stenosis $<50 \%, 37$ with a negative exercise stress test, and only 2 patients with negative perfusion scan were diagnosed as NICP (Fig.1).

In this study median IMA values were definitely higher in patients with ACS compared with NICP ( $p<0.0001)(83.5$ to 49.6). Performance of IMA, cTnT, ECG used alone and in combination with each other for diagnosis of ACS is determined in Table 1; and comparison of sensitivities of them and the differences are illustrated in Figure2.

At presentation IMA diagnosed ACS in 65 of 120 patients ( $54 \%$, CI 51 to 56 ) with chest pain of ischemia origin compared with 105 of 120 (88 \%, CI 83 to 93) by using ECG and 17 of 120 ( $14 \%$, CI 9 to 20 ) by admission cTnT. When IMA was used with ECG in combination the sensitivity rose to $97.5 \%$ (CI 94 to 99 ) and also we have increase in sensitivity by using IMA and cTnT of $48 \%$ (CI 45 to 51 ). Sensitivity and specificity of three tests combined were $97.5 \%$ (CI 94 to 99) and $64.2 \%$ (CI 61 to 65) which is significantly higher than that of IMA and cTnT combined and statistically were near to the sensitivity of IMA and ECG combined. When ECG and cTnT were used $88 \%$ patients are identified. Logistic regression indicates that if IMA is added to conventional diagnostics at ED presentation it doesn't increase the area under the receiver operator characteristic (ROC) curve significantly, from 0.79, using only ECG and cTnT, to 0.80 (Tab. 1).

\section{Discussion}

In the present study, we assessed IMA in patients with ACS and NICP who presented to the emergency room. Our aim was to differentiate transient myocardial ischemia from non-cardiac causes of chest pain. We found that assessment of IMA concentrations in samples obtained from patients at ER admission had $54 \%$ sensitivity and $87 \%$ specificity for diagnosis of ACS. In our study 65 (53\%) of patients with ACS had IMA above $85 \mathrm{U} / \mathrm{ml}$, but patients with NICP 14 (13\%) had abnormal IMA, this finding is similar to that reported by Abadie and colleagues (21) and supports the notion that IMA levels can be used to distinguish myocardial ischemia from non- myocardial ischemia. The main results of our study show that the test of IMA has high specificity of 87 $\%$ compared to study of Hjortshoj and colleagues that had $49 \%$ specificity of this test (11). There have been confusing results regarding IMA and its value in the diagnosis of ACS. Some studies have found the test of possible use, and in particular, a high NPV has attracted attention $(22-25,13)$. However, we found an NPV of only $0.62 \%$ (95\% CI, $0.59-0.66)$ which means that one cannot safely rely on a negative IMA as a single test for excluding ACS.

The limitations of this study are:

1. Absence of normal control group for evaluation of IMA level in normal population.

2. We tested the IMA in ACS patients, however as a proposed ischemia marker, IMA must be able to detect the severest forms of ischemic heart disease.

3. We did not perform gold standard test (angiography) in all patients, which might change our patient's categories and results.

In conclusion, in our population of chest pain patients, we did not find the data supportive of IMA as a standard marker, superior to ECG changes and troponin levels in the emergency department.

\section{References}

1. Ting HH, Lee TH, Soukup JR, Cook EF, Tosteson AN, Brand DA et al. Impact of physician experience on triage of emergency room pitals. Am J Med 1991; 91: 401-408. 
2. Collinson PO, Premachandran S, Hashemi K. Prospective audit of incidence of prognostically important myocardial damage in patients discharged from emergency department. N Engl J Med 2000; 342: 163-167.

3. Storrow AB, Gibler WB, chest pain centers: diagnose of acute coronary syndromes. Ann Emerge Med 2000; 35: 449-461.

4. US Department of Health and Human Services. C.F.D.C.ap. National center for health statics, Hospital discharge data 2002.

5. Pope JH, Aufderheide TP, Ruthazer R, et al. Missed diagnosis of acute cardiac ischemia in the emergency department. N Engl J Med 2000; 342: $1163-7110$.

6. Mc Carthy BD, Beshansky JR, D Agostino RB, Selker HP. Missed diagnoses of acute myocardial infarction in the emergency department: results from a multicenter study. Ann Emerg Med 1993; 22: 579-582.

7. Wu AH, Apple FS, Gibler WB, Jesse RL, Warshaw MM, Valdes R Jr. National Academy of Clinical Biochemistry Standards of Laboratory Practice: recommendations for the use of cardiac markers in coronary artery diseases. Clin Chem 1999; 45: 1104-1121.

Bertrand ME, Simons ML, Fox KA, Wallentin LC, Hamm CW, Mc Fadden et al. Management of acute coronary syndromes in patients presenting without persistent ST-segment elevation. Eur Heart J 2002; 23: 1809-1840.

8. Apple FS, Wu AH, Mair J, Ravkild J, Panteghini M, Tate J, et al. Future biomarkers for detection of ischemia and risk stratification in acute coronary syndrome. Clin Chem 2005; 51 (5): 810-824.

9. Wudkowska A, Goch J, Goch A. Ischemia modified albumin in differential diagnosis of acute coronary syndrome without ST elevation and unstable angina pectoris. Kardiol Pol 2010; 68 (4): 431-437.

10. Hjortshoj S, Risom Kristensen S, Ravkild J. Diagnostic value of ischemia modified albumin in patients with suspected acute coronary syndrome. Am J Emerg Med 2010; 28: 170-176.

11. C Gaze D, Department of chemical pathology .Ischemia Modified Albumin: A Novel Biomarker for the detection of cardiac ischemia. Drug Metab Pharmacokinet 2009; 24 (4): 333-341.

12. Sinha MK, Roy D, C Gaze D, Collinson Po, Kaski Jc. Role of "ischemia modified albumin" a new biochemical marker of myocardial ischemia, in the early diagnosis of acute coronary syndromes. Emerg Med J 2004; 21: 29-34.

13. Chan B, Dodworth N, Woodrow J, Tucker A, Harris R. Site-specific N-terminal auto-degradation of human serum. Eur J Biochem 1995; 227 (1-2): 524-528.
14. McCord J, M: Oxygen derived free radicals in post ischemic tissue injury. N Eng J Med 1985; 312 (3): 159-163.

15. Levine R L: Ischemia from acidosis to oxidation. FASEB J 1993; 7 (13): 1242-1246.

16. Berenshtein E, Mayer B, Goldberg C et al. Patterns of mobilization of copper and Iron following myocardial ischemia. Mol Cell Cardiol J 1997; 29 (11): 3025-3034.

17. HeesChen C, Hamm CW, Bruemmer J, Simoons ML. Investigators: Predictive value of $\mathrm{C}$-reactive protein and troponin $\mathrm{T}$ in patients with unstable angina. A comparative analysis. J Am Coll Cardiol 2000; 35 (6): 1535-1542.

18. Braunwald E, Antom EM, Beasley JW, et al. ACC/AHA guidline update for the management of patients with unstable angina and non-ST segment elevation myocardial infarction. Circulation 2000; 106: 1893-1900.

19. Bar-Or D, Lau E, Winkler JV. A novel assay for cobalt-albumin binding and its potential as a marker for myocardial ischemia - a preliminary report. J Emerg Med 2000; 19: 311-315.

20. Abadie JM, Blassingame CL, Bankson DD. Albumin cobalt binding assay to rule out acute coronary syndrome. Ann Clin Lab Scr 2005; 35: $66-72$.

21. Christenson RH, Duh SH, Sanhai WR, Holtman V, Painter P, Branham E. Characteristics of an albumin cobalt test for assessment of acute coronary syndrome patients: a multicenter study. Clin Chem 2001; 47 (3): 464-470.

22. Collinson PO, Gaze DC, Bainbridge K, Morris F, Morris B, Price A. Utility of admission cardiac troponin and "ischemia modified albumin" measurements for rapid evaluation and rule out of suspected acute myocardial infarction in the emergency department. Emerg Med J 2006; 23 (4): 256-261.

23. Roy D, Quiles J, Aldama G. Ischemia modified albumin for the assessment of patients presenting to the emergency departmentwith acute chest pain but normal or non-diagnostic 12-lead electrocardiograms and negative cardiac troponin T. Int J Cardiol 2004; 97 (2): 297-301.

24. Wu AH, Morris DL, Fletcher DR, Apples Fc, Christenson RH. Analysis of the albumin cobalt binding (ACB) test as an adjunct to cardiac troponin I for the early detection of acute myocardial infarction. Cardiovasc Toxicol 2001; 1 (2): 147-151.

Received January 30, 2011. Accepted June 26, 2012. 